\title{
Electromagnetic polarizabilities of the nucleon and properties of the $\sigma$-meson pole contribution
}

\section{Schumacher ${ }^{\mathrm{a}}$}

Zweites Physikalisches Institut der Universität Göttingen, Friedrich-Hund-Platz 1, D-37077 Göttingen, Germany

Original article: Eur. Phys. J. A 30, 413-422 (2006) DOI: 10.1140/epja/i2006-10103-0

Received: 16 March 2007

Published online: 29 March 2007 - (c) Società Italiana di Fisica / Springer-Verlag 2007

We publish herewith the correct form of eq. (16):

$$
|M(P \rightarrow \gamma \gamma)|=\frac{\alpha_{e}}{\pi f_{P}} N_{c} \sqrt{2} \frac{a e_{u}^{2}+b e_{d}^{2}+c\left(\hat{m} / m_{s}\right) e_{s}^{2}}{\sqrt{a^{2}+b^{2}+c^{2}}}
$$

with $\hat{m} / m_{s} \approx 1 / \sqrt{2}$ being the ratio of light and strange constituent-quark masses. The subsequent calculations have been based on the present version of eq. (16).

\footnotetext{
a e-mail: mschuma3@gwdg.de
} 